\title{
Design of a prospective, multinational registry to evaluate patients hospitalized with hyponatremia: the HN Registry
}

This article was published in the following Dove Press journal:

Open Access Journal of Clinical Trials

9 August 2013

Number of times this article has been viewed

\author{
Paul J Hauptman' \\ Arthur Greenberg ${ }^{2}$ \\ Joseph G Verbalis ${ }^{3}$ \\ Alpesh Amin ${ }^{4}$ \\ Samuel Sigal ${ }^{5}$ \\ Jun Chiong ${ }^{6}$ \\ Sandra Chase ${ }^{7}$ \\ Joseph Dasta ${ }^{8}$
}

'Saint Louis University School of Medicine, St Louis, MO, USA; ${ }^{2}$ Duke University Medical Center, Durham, NC, USA; ${ }^{3}$ Georgetown University Medical Center, Washington, DC, USA; ${ }^{4}$ University of California, Irvine, CA, USA; ${ }^{5}$ New York University Langone Medical Center, New York, NY, USA; 'Loma Linda University, Loma Linda, CA, USA; ${ }^{7}$ Otsuka America Pharmaceutical, Inc, Princeton, NJ, USA; ${ }^{8}$ University of Texas at Austin, TX, USA
Correspondence: Joseph Dasta The University of Texas College of Pharmacy, PO Box 967, Hutto, TX 78634-0967, USA

$\mathrm{Tel}+|5| 2514|82|$

Fax $+|5| 25|4| 82 \mid$

Email jdasta@mail.utexas.edu
Background: Hyponatremia is a prevalent condition in patients hospitalized across a broad range of conditions, including heart failure, cirrhosis, and the syndrome of inappropriate antidiuretic hormone (SIADH) secretion. Whether present on admission or developing during hospitalization, hyponatremia has been associated with increased mortality, longer hospital stays, and higher costs. Little is known, however, about its management and outcomes outside of clinical trial settings.

Methods: The Hyponatremia Registry (HN Registry) is a prospective, observational, multicenter, multinational study of patients hospitalized with either hypervolemic hyponatremia (cirrhosis and heart failure) in the United States or euvolemic hyponatremia (SIADH) in both the United States and Europe. Study enrollment began in September 2010 at community, tertiary, and academic medical centers. Overall, the HN Registry is expected to enroll $>5,000$ patients with hyponatremia, at $>280$ sites. Data will be used to characterize demographic and clinical characteristics of patients hospitalized with hyponatremia, evaluate the comparative effectiveness of available treatment modalities, and document and compare length of hospital stay as a reflection of resource use associated with hospital management.

Discussion: Despite better understanding of the clinical consequences, economic impact, and prognostic significance of euvolemic and hypervolemic hyponatremia, there remains a need to evaluate current "real-world" management. The HN Registry is designed to provide contemporary data on in-hospital evaluation, management, and length of stay in a large cohort of adult patients with hyponatremia. The HN Registry generated several design and analytical challenges that required unique approaches to facilitate collection of the most clinically relevant data.

Keywords: hypervolemia, euvolemia, methodology, design, registry

\section{Introduction}

Hyponatremia, the most common electrolyte abnormality of hospitalized patients, ${ }^{1,2}$ has been associated with increased morbidity, mortality, and resource use, when present at hospital admission or acquired during hospitalization. ${ }^{3-9}$ The management of hyponatremia and its effect on outcomes in clinical practice are, however, poorly understood and have not been critically examined. ${ }^{10,11}$ Defined in many studies as a serum sodium concentration $\left[\mathrm{Na}^{+}\right]<135 \mathrm{mmol} / \mathrm{L}$, the precise frequency of hyponatremia depends on the serum $\left[\mathrm{Na}^{+}\right]$used as the cutoff, the patient population under study, and the clinical setting. Classification based on etiology and appropriate selection of therapeutic options requires an accurate determination of the underlying extracellular fluid volume status; data from small studies suggest that errors in the diagnosis of hyponatremia are common. ${ }^{10,11}$ However, evaluation of contemporary practice in an observational 
registry (especially when the population under study is heterogeneous) is challenging, and issues encountered in the design and implementation of the Hyponatremia Registry (HN Registry) are discussed in this article.

\section{Prevalence}

The prevalence of hyponatremia depends on the threshold serum $\left[\mathrm{Na}^{+}\right]$value selected and the associated disease states and location (eg, acute care facility or outpatient setting) of the population included in the analysis. For example, in a prospective cohort of 98,411 hospitalized adults, a serum $\left[\mathrm{Na}^{+}\right]<135 \mathrm{mmol} / \mathrm{L}$ was found at admission in $14.5 \%$ of patients and developed during hospitalization in an additional $5.2 \% .{ }^{3}$ Other studies have found hyponatremia at admission in up to $30 \%$ of patients. ${ }^{12}$ When more restrictive definitions of hyponatremia are used, the frequency is lower: $\sim 0.5 \%-2.5 \%$ of patients have $\left[\mathrm{Na}^{+}\right]$concentrations $<125 \mathrm{mmol} / \mathrm{L} .^{3,12-14} \mathrm{The}$ occurrence of hyponatremia is higher in older than in younger individuals, ${ }^{14}$ likely due in part, to the increased frequency of comorbid conditions, use of medications associated with hyponatremia, and an idiopathic form of the syndrome of inappropriate antidiuretic hormone secretion (SIADH) found in elderly patients. ${ }^{15}$

\section{Prognostic implications}

In a large retrospective, multicenter study, the presence of a serum $\left[\mathrm{Na}^{+}\right]<135 \mathrm{mmol} / \mathrm{L}$ at hospital admission was independently associated with increased in-hospital mortality and intensive care unit (ICU) admission, of 55\% and $60 \%$, respectively. Hyponatremia was also associated with a 1-day increase in average hospital length of stay (LOS) and an average increase of \$2,289 (in 2005 USD) in total costs/patient. ${ }^{4}$ In other large cohorts, risk of in-hospital mortality, likelihood of ICU admission, hospital LOS, and total costs increased in association with decreasing serum $\left[\mathrm{Na}^{+}\right]$. These adverse outcomes were more frequent even when serum $\left[\mathrm{Na}^{+}\right]$was in the range of 130-134 mmol/L. ${ }^{3,5,16}$ Furthermore, worsening of hyponatremia during hospitalization is associated with increased adjusted odds of in-hospital mortality ${ }^{17}$ and discharge to short- or long-term care facilities. ${ }^{5}$

\section{Treatment}

The treatment of hyponatremia is based on the presence and severity of symptoms, the extracellular volume status of the patient, and whether the onset of hyponatremia was acute ( $<48$ hours) or chronic ( $\geq 48$ hours). ${ }^{18,19}$

Fluid restriction is typically used in less symptomatic cases of euvolemic and hypervolemic hyponatremia; if fluid intake is reduced to a level below the sum of insensible losses and any renal electrolyte-free water excretion, negative water balance will ensue. ${ }^{19,20}$ The degree of water restriction required to increase serum $\left[\mathrm{Na}^{+}\right]$depends on the magnitude of the renal diluting impairment, but restriction to $\leq 1000 \mathrm{~mL} / \mathrm{d}$ is often necessary. From a practical perspective, significant water restriction is difficult to initiate and maintain over long periods and may be relatively ineffective. In general, the success rate for fluid restriction decreases with higher urine osmolality and reduced electrolyte-free water clearance, which reflect higher arginine vasopressin (AVP) concentrations. ${ }^{19,21,22}$

Several drugs have traditionally been used after failure of fluid restriction, including demeclocycline, urea, and lithium. ${ }^{19}$ None of these treatments is approved by the US Food and Drug Administration for use in hyponatremia, and each has limitations. ${ }^{23-31}$ Ultrafiltration has been suggested as an option for treatment of hyponatremia in patients with congestive heart failure, ${ }^{31}$ but this approach extracts isotonic fluid from blood, does not directly improve serum $\left[\mathrm{Na}^{+}\right],{ }^{32}$ and is not approved for the treatment of hyponatremia.

The vasopressin receptor antagonists, or "vaptans," are the newest pharmacologic option for euvolemic or hypervolemic hyponatremia. These agents block the actions of AVP at vasopressin $\mathrm{V}_{2}$ receptors in cells of the renal collecting duct and provide a targeted approach to treatment in patients whose hyponatremia is caused by inappropriately elevated AVP concentrations. ${ }^{19}$ Conivaptan (Astellas Pharma Inc, Tokyo, Japan) is a dual $\mathrm{V}_{1 \mathrm{~A}} / \mathrm{V}_{2}$-receptor antagonist that is available for intravenous use; tolvaptan (Otsuka Pharmaceutical Co, Naruto, Japan) is a selective $\mathrm{V}_{2}$-receptor antagonist that is taken orally once daily and may be continued following hospital discharge. . $^{33,34}$

Given these varying approaches and lack of national guidelines, the multinational observational HN Registry has been designed to provide insight into contemporary practice and clinical response to medical interventions across most euvolemic and hypervolemic hyponatremic patients. The data collected from the HN Registry will provide important analyses of the contemporary use of various diagnostic and therapeutic approaches, including duration of therapy, effect on serum $\left[\mathrm{Na}^{+}\right]$, and impact on LOS.

\section{HN registry methods}

The HN Registry (ClinicalTrials.gov Identifier: NCT01240668) is a prospective, observational, multicenter study of patients hospitalized with euvolemic or hypervolemic hyponatremia in the United States and with hyponatremia 
secondary to SIADH in seven European countries (Denmark, France, Germany, Italy, Spain, Sweden, and the United Kingdom). The HN Registry was designed to provide "realworld" data on patients with serum $\left[\mathrm{Na}^{+}\right] \leq 130 \mathrm{mmol} / \mathrm{L}$, excluding patients with hypovolemic hyponatremia. Although hyponatremia is commonly defined as a serum $\left[\mathrm{Na}^{+}\right]<135 \mathrm{mmol} / \mathrm{L}$, a cut-off value of $\leq 130 \mathrm{mmol} / \mathrm{L}$ was chosen to select patients with increased risk for clinical symptoms of hyponatremia and greater likelihood of receiving therapies intended to correct low serum $\left[\mathrm{Na}^{+}\right]$.

The specific, predefined objectives of the HN Registry are to: (1) obtain demographic and clinical characteristics of patients with euvolemic or hypervolemic hyponatremia in the hospital setting; (2) evaluate the effectiveness of prescribed treatment modalities in these patients; and (3) define and compare LOS as a surrogate for resource usage associated with hospital management.

Patients are identified by study personnel based on serum $\left[\mathrm{Na}^{+}\right]$; criteria have been established to minimize the likelihood of inclusion of patients with hypovolemic hyponatremia. Following an extensive training session, site study coordinators extract data from the inpatient medical record, starting with hospital admission and continuing through discharge. The data are entered in a case report form or electronic data capture system. Because this is an observational registry, both diagnosis and treatment are determined by the patient's treating physicians and not by HN Registry investigators.

The study is being conducted in compliance with all national and local regulatory requirements. The study protocol must be approved by the institutional review board or independent ethics committee at each hospital, before patient enrollment is initiated. In Europe, as required, the approval of the appropriate national and/or regional regulatory bodies was also obtained. When a waiver of consent is not granted by the appropriate ethics committee, individual informed patient consent is obtained prior to data collection.

Study enrollment began in September 2010 at community, tertiary, and academic medical centers, with expected enrollment of 5,000 patients, from $>280$ institutions. The clinical research organization providing support is REGISTRAT-MAPI (Lyon, France). Funding is provided by Otsuka America Pharmaceutical, Inc (Rockville, MD, USA).

\section{Inclusion and exclusion criteria}

The inclusion and exclusion criteria are shown in Table 1. Briefly, hospitalized adults aged $\geq 18$ years are eligible if they have euvolemic (United States and Europe) or
Table I Inclusion and exclusion criteria

\section{Inclusion criteria}

I. Adults aged $\geq 18$ years who are hospitalized

2. Euvolemic or hypervolemic hyponatremia with serum $\left[\mathrm{Na}^{+}\right] \leq 130 \mathrm{mmol} / \mathrm{L}$

3. For euvolemic hyponatremia:

- Euvolemia is defined as absence of clinical and historical evidence of extracellular fluid volume depletion or sequestration and absence of edema and ascites; or

- Physician diagnosis of SIADH

4. For hypervolemic hyponatremia (applies to US sites only):

- Hypervolemia is defined as excess extracellular fluid volume manifesting as dependent edema or ascites

- Patients may have $\geq \mathrm{I}$ of the following underlying comorbid conditions:

- Congestive heart failure

- Cirrhosis and/or liver failure

o Nephrotic syndrome

\section{Exclusion criteria}

I. Patients with hypovolemic hyponatremia

2. Use of any investigational drug, biologic, or device during the study

3. Random blood glucose $>250 \mathrm{mg} / \mathrm{dL}$, or between 180 and $250 \mathrm{mg} / \mathrm{dL}$ with serum $\left[\mathrm{Na}^{+}\right]$of $127-130 \mathrm{mmol} / \mathrm{L}$ at entry

4. Patient receiving renal replacement therapy for chronic kidney disease or acute kidney injury

Abbreviations: $\left[\mathrm{Na}^{+}\right]$, sodium concentration; SIADH, syndrome of inappropriate antidiuretic hormone.

hypervolemic (United States only) hyponatremia characterized by a serum $\left[\mathrm{Na}^{+}\right] \leq 130 \mathrm{mmol} / \mathrm{L}$ either on admission or developing during their hospital stay. The chart-based diagnosis of euvolemia requires the absence of clinical or historical evidence of extracellular fluid volume expansion or depletion, including the absence of edema and ascites, or a physician's written diagnosis of SIADH. In addition, the urine $\left[\mathrm{Na}^{+}\right]$should not be $<20 \mathrm{mmol} / \mathrm{L}$ (if measured), and the blood urea nitrogen (BUN):creatinine ratio should not be $>20$ in patients listed as euvolemic. Hypervolemia is defined as excess extracellular fluid volume manifesting as dependent edema or ascites in the presence of $\geq 1$ comorbid condition of congestive heart failure, cirrhosis, liver failure, or nephrotic syndrome.

Key exclusion criteria include hypovolemic hyponatremia and random blood glucose $>250 \mathrm{mg} / \mathrm{dL}$, or $180-250 \mathrm{mg} / \mathrm{dL}$ together with serum $\left[\mathrm{Na}^{+}\right]$of $127-130 \mathrm{mmol} / \mathrm{L}$ at entry. Patients receiving renal replacement therapy for either chronic kidney disease or acute kidney injury are also excluded. Patients may not be enrolled if they are simultaneously receiving an investigational drug or other agent in a clinical trial setting.

\section{Data collection}

Data collected on hospital admission include date of hospitalization, admitting diagnosis, demographics (age, sex, 
and in the United States only, race), details on the underlying condition (including left-ventricular ejection fraction and New York Heart Association classification for heart failure, ${ }^{35}$ Child-Pugh classification ${ }^{36}$ and Model for End-Stage Liver Disease score for cirrhosis, ${ }^{37}$ and underlying etiology for SIADH), history of hyponatremia (including number of prior hospitalizations in the past year and acuity of onset of hyponatremia, when available), medications at hospital admission (including those prescribed to treat hyponatremia, natriuretic diuretics, and hyponatremia-inducing drugs), and vital signs at admission and on the day hyponatremia was first documented.

Additional data are collected from the patient's medical record on each day of hospitalization, including body weight, signs and symptoms potentially associated with hyponatremia, serum $\left[\mathrm{Na}^{+}\right]$, and other standard laboratory tests, such as serum electrolytes. If obtained, results of other relevant laboratory tests will be recorded, including urinary electrolytes, urinary creatinine and urea nitrogen, serum and urine osmolality, thyroid-stimulating hormone, cortisol, results of a cosyntropin stimulation test, and heart failure biomarkers (ie, B-type natriuretic peptide and N-terminal pro-B-type natriuretic peptide). Documentation also includes volume of fluid intake and output over each 24-hour period (if available); information on the amount and duration of fluid restriction, including days on which adjustments in the degree of fluid restriction are made; details pertaining to treatment with intravenous saline, including type (hypertonic 2\%-3\% or isotonic $0.9 \%$ solution); medications used to treat hyponatremia; natriuretic diuretics and other hyponatremia-inducing drugs; procedures used to manage comorbidities; specialty of the physician responsible for the patient's management during the hospital stay and use of subspecialty consultations; and hospital LOS, including LOS in ICU and intermediate care unit. At the time of hospital discharge, data capture includes discharge medications and disposition.

\section{Endpoints and endpoint adjudication}

The analysis of study endpoints is descriptive and associative, consistent with the observational design of the study. The HN Registry has two primary endpoints: change in serum $\left[\mathrm{Na}^{+}\right]$from the beginning to the end of the treatment period or hospital discharge and hospital LOS from time of first presentation of hyponatremia (day of admission or subsequently) to discharge.

Secondary endpoints include the effectiveness of individual therapies, alone and in combination, for correction of hyponatremia, and time needed to achieve correction, as well as the effectiveness of individual therapies in achieving symptom improvement. Given the inability of a registry-based study to impose a standardized protocol on treating physicians or mandate achievement of a particular treatment goal, we prespecified several broad definitions of correction: achievement of a serum $\left[\mathrm{Na}^{+}\right]>130 \mathrm{mmol} / \mathrm{L}$, a serum $\left[\mathrm{Na}^{+}\right] \geq 135 \mathrm{mmol} / \mathrm{L}$, and/or an increase in serum $\left[\mathrm{Na}^{+}\right]$by $\geq 5 \mathrm{mmol} / \mathrm{L}$. If the serum $\left[\mathrm{Na}^{+}\right]$initially corrects to a value $>130 \mathrm{mmol} / \mathrm{L}$ but subsequently falls below that threshold, this new "episode" is analyzed as a separate casebased event.

Secondary resource usage endpoints include medically necessary LOS in hospital and specialized hospital units, and impact of hyponatremia on ICU and overall hospital LOS. The study is also designed to evaluate several descriptive secondary endpoints, including the relative proportions of underlying etiologies of hyponatremia, the investigations performed to diagnose hyponatremia, and time to treatment initiation and type of therapies used to treat hyponatremia.

Cases that do not appear to meet inclusion, exclusion, or secondary criteria (Table 2), based on initial evaluation by the HN Registry project manager, are reviewed in a separate adjudication process performed independently by $\geq 2$ physician members of the HN Registry Steering Committee. This mechanism was established to ensure that inclusion and exclusion criteria are satisfied; patients who fail to meet eligibility criteria will be considered as screen failures and will be dropped from the analysis (see Steering Committee participation).

In addition, multiple means of analyzing LOS data will be used to mitigate the challenge of confounders, such as

Table 2 Criteria used to refer patients for adjudication

I. $<24$ hours of serum $\left[\mathrm{Na}^{+}\right] \leq 130 \mathrm{mmol} / \mathrm{L}$

2. Correction of serum $\left[\mathrm{Na}^{+}\right](>/ 30 \mathrm{mmol} / \mathrm{L})$ after administration of isotonic saline as principal therapy

3. Random blood glucose $>250 \mathrm{mg} / \mathrm{dL}$, or between 180 and $250 \mathrm{mg} / \mathrm{dL}$ with serum $\left[\mathrm{Na}^{+}\right]$of $127-130 \mathrm{mmol} / \mathrm{L}$

4. Patients receiving renal replacement therapy

5. Urine $\left[\mathrm{Na}^{+}\right]<20 \mathrm{mmol} / \mathrm{L}$ in ostensibly euvolemic patients

6. Patients presenting with cerebral salt wasting

7. BUN:creatinine ratio $>20$ for euvolemic and $>40$ for hypervolemic patients

8. Diagnosis of heart failure and euvolemia

9. Diagnosis of cirrhosis and euvolemia

10. Diagnosis of nephrotic syndrome and euvolemia

II. Diagnosis of SIADH and hypervolemia

12. Diagnosis of hypervolemia or euvolemia, but no comorbidity or etiology described

Abbreviations: BUN, blood urea nitrogen; $\left[\mathrm{Na}^{+}\right]$, sodium concentration; $\mathrm{SIADH}$, syndrome of inappropriate antidiuretic hormone. 
severity of patient illness or prolonged LOS due to nonmedical reasons (see Design challenges).

\section{Steering Committee participation}

The seven-member Steering Committee comprising two cardiologists, a nephrologist, an endocrinologist, a hospitalist, a hepatologist, and a pharmacist was formed and chartered to develop the protocol, advise on scientific and logistical issues, and monitor the progress of enrollment. In addition, physician Steering Committee members engage in a formal adjudication process for the subset of cases that appear on initial screening to require additional scrutiny before inclusion in the HN Registry database. Excluded cases include those in which the serum $\left[\mathrm{Na}^{+}\right]$ was $\leq 130 \mathrm{mmol} / \mathrm{L}$ for a duration $<24$ hours and when the diagnosis of heart failure or cirrhosis was accompanied by the diagnosis of euvolemia.

The sponsor plays a collaborative role, but the Steering Committee supervises the formal adjudication process and makes final decisions about patient inclusion.

\section{Design challenges}

The present investigation, subject to the inherent limitations of any registry, presented a number of design challenges that had to be overcome (Table 3).

Ideally, a registry should enroll consecutive patients. Doing so permits accrual of data about prevalence of various etiologies of hyponatremia and eliminates selection bias. However, for hyponatremia, consecutive enrollment would require ascertainment by screening all cases identified by the laboratory, with serum $\left[\mathrm{Na}^{+}\right]$below the inclusion threshold. Because we were interested in tracking management methods employed by a variety of specialists, the roster of investigators was not limited to endocrinologists and nephrologists to whom unselected hyponatremia cases might be referred.

\section{Table 3 Challenges in the design of the HN Registry}

I. Development of criteria for hypovolemia, euvolemia, and hypervolemia

2. Definition of "correction" of serum $\left[\mathrm{Na}^{+}\right]$

3. Definition of a new "episode" of hyponatremia if serum $\left[\mathrm{Na}^{+}\right]$ initially corrects, but subsequently redevelops later in hospitalization

4. Accounting for confounders that impact LOS

5. Developing various LOS calculations based on time of identification and treatment of hyponatremia ${ }^{39}$

6. Identifying symptoms attributed to hyponatremia and their improvement based on documentation in medical record

7. Developing strategies to identify patients for enrollment

8. Avoidance of selection bias in an observational study design

Abbreviations: $\mathrm{HN}$, hyponatremia; $\mathrm{LOS}$, length of stay; $\left[\mathrm{Na}^{+}\right]$, sodium concentration.
Rather, we also recruited cardiologists and hepatologists, whose practice and patient accrual are likely limited to patients with primary disorders within their specialty. This choice means we can report the distribution of etiologies among enrolled patients but not the distribution among unselected hyponatremic patients in general. However, we did encourage investigators to enroll consecutive patients, as a means of minimizing bias.

The customary definition of SIADH is euvolemic hypotonic hyponatremia occurring due to water retention induced by persistent secretion of vasopressin not fully suppressed despite hypotonicity. Patients must be euvolemic by conventional clinical assessment, with no evidence of volume depletion and no edema. Confirmatory laboratory criteria include a documented low serum osmolality with non-maximally dilute urine and normal thyroid and adrenal function. In the HN Registry, no specific protocol for diagnosis of hypervolemic or euvolemic hyponatremia was imposed on the study centers because the major goal was to determine which diagnostic tests and procedures were chosen by clinicians as they managed hyponatremia. In the absence of a standardized evaluation, one of the prevalent limitations identified during the early phases of data collection was the frequent lack of sufficient detail in the medical record to categorize volume status and determine the precise etiology of hyponatremia. We addressed this issue by abstracting urine sodium concentration, BUN:creatinine ratio, and response to normal saline infusion, if present in the medical record, to confirm or refute the absence of volume depletion. Correction of hyponatremia after normal saline infusion suggests that volume depletion was present and was the cause of the hyponatremia. Patients classified as having euvolemic hyponatremia who had a BUN:creatinine ratio $>20: 1$, a urine sodium $<20 \mathrm{mmol} / \mathrm{L}$, or whose hyponatremia was corrected solely by administration of isotonic saline infusion were referred for adjudication to confirm that the diagnosis of euvolemia was correct. In addition, no specific goal for posttreatment serum $\left[\mathrm{Na}^{+}\right]$ can be imposed in the real-world setting. The serum $\left[\mathrm{Na}^{+}\right]$ achieved before a patient is deemed by the treating physicians to be stable enough for discharge is expected to vary with baseline serum $\left[\mathrm{Na}^{+}\right]$and status of the underlying disease. As a result, the three separate definitions of correction (vide supra) were designated.

Further, because some patients receive multiple and temporally overlapping therapies that could impact serum $\left[\mathrm{Na}^{+}\right]$, attributing response to a single intervention may be difficult. An intention-to-treat approach was considered, but 
fluid restriction is frequently used and often the first-line treatment for hyponatremia, such that the potential impact of second- and third-line therapies used sequentially would not be apparent. We chose to describe which approaches are taken and when, and to monitor the overall rate of correction (or lack thereof) in serum $\left[\mathrm{Na}^{+}\right]$. By extension, another limitation of the HN Registry design is that the order and choice of therapies was at the discretion of the treating clinician rather than stipulated by an experimental plan. A preliminary list of the many treatment maneuvers used in the HN Registry, based on an analysis of the first 500 adjudicated cases, is shown in Table 4. The list includes treatment regimens that were identified in $\geq 1 \%$ of the treatment events. Multiple episodes of hyponatremia may occur during a single patient's hospitalization and, therefore, some patients may be counted more than once. The analytical plan calls for examination of both sequential treatments (patients who receive $\geq 2$ non-overlapping therapies) and combination treatments (patients who receive $\geq 2$ therapies together during a given treatment period).

We limited evaluation of resource utilization to LOS, in part because the collection of hospital bills would mandate informed consent. Nevertheless, it will be difficult to control for confounders such as comorbidities, severity of illness, and nonmedical factors like bed availability at skilled nursing facilities. The absence of reliable and systematic evaluation and documentation of patient symptoms associated with hyponatremia and their subsequent improvement present additional challenges; for example, the attribution of symptoms to hyponatremia may be confounded by coexisting hepatic encephalopathy in cirrhosis or low cardiac output syndrome in heart failure.

Table 4 Treatment maneuvers used in the HN Registry

\begin{tabular}{|c|c|}
\hline Therapy & $\mathbf{N}(\%)$ \\
\hline Fluid restriction & $335(34.1)$ \\
\hline Normal saline & $115(\mathrm{II} .7)$ \\
\hline Fluid restriction and normal saline & $92(9.4)$ \\
\hline Fluid restriction and tolvaptan & $80(8.1)$ \\
\hline Fluid restriction and other therapy ${ }^{a}$ & $64(6.5)$ \\
\hline Tolvaptan ${ }^{b}$ & $62(6.3)$ \\
\hline Other therapy ${ }^{\mathrm{a}}$ & $41(4.2)$ \\
\hline Fluid restriction and hypertonic saline & $30(3.1)$ \\
\hline Fluid restriction, normal saline, and other therapy ${ }^{a}$ & $22(2.2)$ \\
\hline Hypertonic saline & $19(1.9)$ \\
\hline Fluid restriction, normal saline, and tolvaptan & $14(1.4)$ \\
\hline Normal saline and other therapy ${ }^{\mathrm{a}}$ & II (I.I) \\
\hline Fluid restriction, normal and hypertonic saline & $10(1.0)$ \\
\hline
\end{tabular}

Notes: ${ }^{-U}$ rea, salt tablets, demeclocycline; ${ }^{\mathrm{b}}<1 \%$ of patients received conivaptan. Abbreviation: $\mathrm{HN}$, hyponatremia.

\section{Preliminary insights}

A rigorous process of data collection, quality control, and data "scrubbing" has been completed for the first 500 patients in the HN Registry. Preliminary data are shown in Table 5. The mean age is higher than in most randomized controlled trials involving hyponatremia, and LOS is long, consistent with the severity and in most cases, chronicity of the underlying medical diagnoses. Furthermore, the majority of patients within the HN Registry were treated by either hospitalists or internists. Subspecialists, primarily nephrologists and endocrinologists, were consulted for hyponatremia in 211 patients $(42.2 \%)$.

\section{Discussion}

Observational registries can be powerful tools to document the course of disease and variations in treatment and outcomes, and provide valuable insights about real-world medical practice. In particular, registries can provide information regarding the clinical context when multiple or complex therapies are administered and can also generate hypotheses for further study that may not be obtained from randomized controlled trials. ${ }^{38}$ The utility and applicability of registry data, however, rely heavily on the quality of the data obtained and, in particular, the inclusion and exclusion criteria used to define the patient population under study. Because of the nature of their design, randomized controlled trials minimize selection bias to allow comparison between treated and nontreated patients; however, they do not provide information on real-world practice.

The HN Registry, the first study to provide an in-depth understanding of contemporary real-world management practices for euvolemic or hypervolemic hyponatremia, incorporates a rigorous process to define inclusion and exclusion of cases, and thus will allow an evaluation of patient

Table 5 Preliminary insights of first 500 patients

\begin{tabular}{ll}
\hline Variable & $\begin{array}{l}\text { N (\% or SD where } \\
\text { applicable) }\end{array}$ \\
\hline Euvolemic, $\mathrm{n}(\%)$ & $179(35.8)$ \\
Hypervolemic, $\mathrm{n}(\%)$ & $321(64.2)$ \\
SIADH, $\mathrm{n}$ & 176 \\
Congestive heart failure, $\mathrm{n}$ & 168 \\
Cirrhosis, $\mathrm{n}$ & 170 \\
Nephrotic syndrome, $\mathrm{n}$ & 14 \\
>I comorbidity in hypervolemic patients, $\mathrm{n}$ & 23 \\
Median age, (years) & 63 \\
Mean LOS (SD), (days) & $10.1(8.5)$ \\
\hline
\end{tabular}

Note: Patients can have more than one co-morbidity.

Abbreviations: LOS, length of stay; SD, standard deviation; SIADH, syndrome of inappropriate antidiuretic hormone. 
outcomes and current resource usage. Careful consideration was given to the design of the HN Registry, the quality of the collected data, and the analytical approach. Close collaboration between Steering Committee members, statisticians, and study coordinators involved in the development and management of the HN Registry will be instrumental in ensuring quality of the collected data. Preliminary data suggest that a large number of treatment regimens are currently being used by physicians to manage hyponatremia. Ultimately, information from the HN Registry may help to define and refine optimal care for patients hospitalized with hyponatremia or who develop hyponatremia during hospitalization.

\section{Acknowledgment}

The HN Registry is funded by Otsuka America Pharmaceutical, Inc.

\section{Disclosure}

Drs Hauptman, Greenberg, Verbalis, Amin, Sigal, and Chiong, and Prof Dasta are members of the HN Registry Steering Committee and as such, act as paid consultants for Otsuka America Pharmaceutical. However, these authors have not been compensated for preparing this manuscript. Dr Chase is an employee of Otsuka America Pharmaceutical. The authors report no other conflicts of interest.

\section{References}

1. Palmer BF, Gates JR, Lader M. Causes and management of hyponatremia. Ann Pharmacother. 2003;37(11):1694-1702.

2. Schrier RW, Bansal S. Diagnosis and management of hyponatremia in acute illness. Curr Opin Crit Care. 2008;14(6):627-634.

3. Waikar SS, Mount DB, Curhan GC. Mortality after hospitalization with mild, moderate, and severe hyponatremia. Am J Med. 2009;122(9):857-865.

4. Zilberberg MD, Exuzides A, Spalding J, et al. Epidemiology, clinical and economic outcomes of admission hyponatremia among hospitalized patients. Curr Med Res Opin. 2008;24(6):1601-1608.

5. Wald R, Jaber BL, Price LL, Upadhyay A, Madias NE. Impact of hospital-associated hyponatremia on selected outcomes. Arch Intern Med. 2010;170(3):294-302.

6. Gheorghiade M, Rossi JS, Cotts W, et al. Characterization and prognostic value of persistent hyponatremia in patients with severe heart failure in the ESCAPE Trial. Arch Intern Med. 2007;167(18):1998-2005.

7. Shorr AF, Tabak YP, Johannes RS, Gupta V, Saltzberg MT, Costanzo MR. Burden of sodium abnormalities in patients hospitalized for heart failure. Congest Heart Fail. 2011;17(1):1-7.

8. Doshi SM, Shah P, Lei X, Lahoti A, Salahudeen AK. Hyponatremia in hospitalized cancer patients and its impact on clinical outcomes. Am J Kidney Dis. 2012;59(2):222-228.

9. Zilberberg MD, Exuzides A, Spalding J, et al. Hyponatremia and hospital outcomes among patients with pneumonia: a retrospective cohort study. BMC Pulm Med. 2008;8:16.

10. Huda MS, Boyd A, Skagen K, et al. Investigation and management of severe hyponatraemia in a hospital setting. Postgrad Med J. 2006;82(965):216-219.
11. Whyte M, Down C, Miell J, Crook M. Lack of laboratory assessment of severe hyponatraemia is associated with detrimental clinical outcomes in hospitalised patients. Int J Clin Pract. 2009;63(10): 1451-1455.

12. Hoorn EJ, Lindemans J, Zietse R. Development of severe hyponatraemia in hospitalized patients: treatment-related risk factors and inadequate management. Nephrol Dial Transplant. 2006;21(1):70-76.

13. Hawkins RC. Age and gender as risk factors for hyponatremia and hypernatremia. Clin Chim Acta. 2003;337(1-2):169-172.

14. Upadhyay A, Jaber BL, Madias NE. Incidence and prevalence of hyponatremia. Am J Med. 2006;119(7 Suppl 1):S30-S35.

15. Hirshberg B, Ben-Yehuda A. The syndrome of inappropriate antidiuretic hormone secretion in the elderly. Am J Med. 1997;103(4): 270-273.

16. Callahan MA, Do HT, Caplan DW, Yoon-Flannery K. Economic impact of hyponatremia in hospitalized patients: a retrospective cohort study. Postgrad Med. 2009;121(2):186-191.

17. Gill G, Huda B, Boyd A, et al. Characteristics and mortality of severe hyponatraemia - a hospital-based study. Clin Endocrinol (Oxf). 2006;65(2):246-249.

18. Sterns RH, Cappuccio JD, Silver SM, Cohen EP. Neurologic sequelae after treatment of severe hyponatremia: a multicenter perspective. $J \mathrm{Am}$ Soc Nephrol. 1994;4(8):1522-1530.

19. Verbalis JG, Goldsmith SR, Greenberg A, Schrier RW, Sterns RH. Hyponatremia treatment guidelines 2007: expert panel recommendations. Am J Med. 2007;120(11 Supp1 1):S1-S21.

20. Androgué HJ, Madias NE. Hyponatremia. N Engl J Med. 2000; 342(21):1581-1589.

21. Furst H, Hallows KR, Post J, et al. The urine/plasma electrolyte ratio: a predictive guide to water restriction. Am J Med Sci. 2000;319(4): 240-244.

22. Berl T. Impact of solute intake on urine flow and water excretion. J Am Soc Nephrol. 2008;19(6):1076-1078.

23. Sherlock M, Thompson CJ. The syndrome of inappropriate antidiuretic hormone: current and future management options. Eur J Endocrinol. 2010;162 Suppl 1:S13-S18.

24. Miller PD, Linas SL, Schrier RW. Plasma demeclocycline levels and nephrotoxicity. Correlation in hyponatremic cirrhotic patients. JAMA. 1980;243(24):2513-2515.

25. Oster JR, Epstein M, Ulano HB. Deterioration of renal function with demeclocycline administration. Curr Ther Res Clin Exp. 1976;20(6): 794-801.

26. Carrilho F, Bosch J, Arroyo V, Mas A, Viver J, Rodes J. Renal failure associated with demeclocycline in cirrhosis. Ann Intern Med. 1977;87(2):195-197.

27. Curtis NJ, van Heyningen C, Turner JJ. Irreversible nephrotoxicity from demeclocycline in the treatment of hyponatremia. Age Ageing. 2002;31(2):151-152.

28. Zegers de Beyl D, Naeije R, de Troyer A. Demeclocycline treatment of water retention in congestive heart failure. $\mathrm{Br}$ Med J. 1978; 1(6115):760.

29. Decaux G, Brimioulle S, Genette F, Mockel J. Treatment of the syndrome of inappropriate secretion of antidiuretic hormone by urea. Am J Med. 1980;69(1):99-106.

30. Goldsmith SR. Current treatments and novel pharmacologic treatments for hyponatremia in congestive heart failure. Am J Cardiol. 2005;95(9A):14B-23B.

31. Dickstein K, Cohen-Solal A, Filippatos G, et al; ESC Committee for Practice Guidelines (CPG). ESC guidelines for the diagnosis and treatment of acute and chronic heart failure 2008: the Task Force for the diagnosis and treatment of acute and chronic heart failure 2008 of the European Society of Cardiology. Developed in collaboration with the Heart Failure Association of the ESC (HFA) and endorsed by the European Society of Intensive Care Medicine (ESICM). Eur J Heart Fail. 2008;10(10):933-989.

32. Kazory A. Haemodialysis, not ultrafiltration, can correct hyponatraemia in heart failure. Eur J Heart Fail. 2010;12(2):208. 
33. VAPRISOL ${ }^{\circledR}$ (conivaptan hydrochloride) injection, for intravenous use [prescribing information]. Northbrook, IL: Astellas Pharma US, Inc; 2012.

34. SAMSCA ${ }^{\mathrm{TM}}$ (tolvaptan) tablets [prescribing information]. Rockville, MD: Otsuka America Pharmaceutical, Inc; 2013.

35. The Criteria Committee of the New York Heart Association. Nomenclature and Criteria for Diagnosis of Diseases of the Heart and Great Vessels. 9th ed. Boston: Little, Brown \& Co; 1994:253-256.

36. Child CG, Turcotte JG. Surgery and portal hypertension. In: CG Child, editor. The liver and portal hypertension. Philadelphia: Saunders; 1964:50-64.
37. Malinchoc M, Kamath PS, Gordon FD, et al. A model to predict poor survival in patients undergoing transjugular intrahepatic portosystemic shunts. Hepatology. 2000;31: 864-71.

38. Nallamothu BK, Hayward RA, Bates ER. Beyond the randomized clinical trial: the role of effectiveness studies in evaluating cardiovascular therapies. Circulation. 2008;118(12):1294-1303.

39. Dasta JF, Amin A, Chiong JR, Greenberg A, Verbalis JG, Chiodo J. Challenges in assessing the impact of hyponatremia management on length of stay: interim results from a global, multi-center, prospective, observational registry of hospitalized hypervolemic and euvolemic hyponatremic patients. Value Health. 2013;16(3):A18.

\section{Publish your work in this journal}

The Open Access Journal of Clinical Trials is an international, peerreviewed, open access journal publishing original research, reports, editorials, reviews and commentaries on all aspects of clinical trial design, management, legal, ethical and regulatory issues, case record form design, data collection, quality assurance and data auditing methodologies. The manuscript management system is completely online and includes a very quick and fair peer-review system, which is all easy to use. Visit http://www.dovepress.com/testimonials.php to read real quotes from published authors.

Submit your manuscript here: http://www.dovepress.com/open-access-journal-of-clinical-trials-journal 